\begin{tabular}{|l|l|}
\hline AB0217 & NON MEDICAL SWITCH FROM ETANERCEPT \\
ORIGINATOR TO BIOSIMILAR GP2015 IN PATIENTS \\
WITH CHRONIC INFLAMMATORY ARTHROPATHIES
\end{tabular}

M. Colaci' ${ }^{1}$ M. L. Aprile ${ }^{1}$, A. La Rosa ${ }^{2}$, A. DI Maggio², L. Malatino ${ }^{1}$ ${ }^{1}$ Rheumatology Centre, Ospedale Cannizzaro, Clinical and Experimental Medicine, University of Catania, Catania, Italy; ${ }^{2}$ Servizio di Farmacia Ospedaliera, Ospedale Cannizzaro, Catania, Italy

Background: In the last decades, new biologic drugs were introduced for the treatment of chronic inflammatory arthropathies, progressively leading to a relevant increase of medical costs. However, the introduction of biosimilars (biologic molecules similar to branded drugs with expired patent) permitted to optimize the financial resources. The non-medical switch (NMS) is the switch from a biologic originator to a biosimilar agent for economic reasons only, on the basis of the substantial equivalence as regards efficacy and safety between originator and biosimilar drugs.

In literature, several evidences from clinical trials and registry studies showed that the switch from etanercept originator to biosimilar SB4 was safe [1]. Instead no sufficient data may be found regarding the biosimilar GP2015.

Objectives: We aimed to evaluate efficacy, safety, and retention rate in a series of patients with chronic arthritis treated with etanercept originator who underwent to NMS towards the ETN biosimilar GP2015.

Methods: From March to June 2020, all patient referred in our Centre affected by rheumatoid arthritis (RA), psoriatic arthritis (PA) and axial spondyloarthritis (axSpA) treated with etanercept originator and in remission/low disease activity for at least 6 months underwent to NMS. Data on disease activity (DAS28-PCR/CDAI/SDAI; DAPSA; BASDAI), eventual adverse events and causes of withdrawal of therapy were collected at 2, 4 and 6 months after the switch.

Results: We recruited 71 consecutive patients (M/F: 24/47; mean age 55,8 11,1 years; 39 RA; 15 PA; 17 axSpA; mean duration therapy $7.3 \pm 3.8$ years). Disease activity was unchanged for almost all patients after 6 months from the switch (median $\triangle \mathrm{DAS} 28-\mathrm{PCR} / \mathrm{CDAl} / \mathrm{SDAl}$ : 0,1/0/0,5; median $\triangle \mathrm{DAPSA}$ : 0; median $\triangle$ BASDAl: 0) Moreover, the 6 -month retention rate was $97.2 \%$. Only 2 patients $(2.8 \%)$ switched back to the originator due to loss of efficacy in one case and adverse events in the second case (paraesthesia, headache, dizziness and worsening of arthralgia).

Conclusion: Our study confirmed that the NMS from ETN originator to GP2015 represents a safe practice that maintains the efficacy of the current treatment. REFERENCES:

[1] Glintborg B, AG, Omerovic E, et Al. To switch or not to switch: results of a nationwide guideline of mandatory switching from originator to biosimilar etanercept. One-year treatment outcomes in 2061 patients with inflammatory arthritis from the DANBIO registry. Ann Rheum Dis 2019; 78:192-200.

Disclosure of Interests: None declared

DOI: 10.1136/annrheumdis-2021-eular.1557

\section{AB0218 NON MEDICAL SWITCH FROM ADALIMUMAB ORIGINATOR TO THE BIOSIMILAR GP2017 IN PATIENTS AFFECTED BY CHRONIC ARTHRITIS}

M. Colaci ${ }^{1}$, M. L. Aprile ${ }^{1}$, A. La Rosa ${ }^{2}$, A. DI Maggio ${ }^{2}$, L. Malatino'. ${ }^{1}$ Rheumatology Centre, Ospedale Cannizzaro, Clinical and Experimental Medicine, University of Catania, Catania, Italy; ${ }^{2}$ Servizio di Farmacia Ospedaliera, Ospedale Cannizzaro, Catania, Italy

Background: The non-medical switch (NMS) from originator drugs to biosimilars is a possible strategy adopted by rheumatologists in order to control the expansion of pharmaceutic costs for the treatment fo patients affected by chronic arthritis. To date, few real life data on the switch from adalimumab (ADA) originator to its biosimilars are available.

Objectives: We aimed to evaluate efficacy and safety in a single-Centre cohort of patients affected by chronic arthritis who switched from ADA originator to the biosimilar GP2017.

Methods: Patients affected by rheumatoid arthritis (RA), psoriatic arthritis (PA) and axial spondyloarthritis (axSpA) already on therapy with ADA originator in remission or low disease activity for at least 6 months were switched to GP2017 (March- June 2020). Data on disease activity (DAS28-PCR/CDAI/SDAI; DAPSA; BASDAI), eventual adverse events and causes of withdrawal of therapy were collected at 2, 4 and 6 months after the switch.

Results: 88 patients were enrolled (M/F 36/52; mean age $55.8 \pm 12$ years; 25 RA, 32 PA, 31 axSpA, mean duration of therapy $6.3 \pm 3.8$ years). No statistically significant difference was observed in median DAS28-PCR/CDAI/SDAI values at the baseline and after 6 months $[1.03(0.96-3.43)$ vs. $1.21(0-3.7)$ / $0(0-15)$ vs. $0(0-17) / 0(0-15)$ vs. $0.5(0-17.5)]$, DAPSA [0 (0-12.2) vs. 0
(0-15)] and BASDAI [0 (0-4.3) vs. $0(0-6.4)]$. The retention rate was $93.2 \%$. $6 / 88$ patients $(6.8 \%)$ switched back to the originator. The causes of discontinuation were: disease reactivation in a single case $(1.1 \%)$, subjective reasons/ nocebo effect in 5/88 cases (5.7\%), including: general malaise and transient increased of blood pressure (n.1), dizziness, paraesthesia, arthralgia, headache (n.1), itch sine materia (n.1) and subjective worsening without objective disease flare (n.2).

Conclusion: This is the first real life study showing that the NMS from ADA originator to GP2017 represents a safe practice that maintains the efficacy of the current treatment. However, a few cases of switchback were described, mainly attributed to nocebo effect [1]

\section{REFERENCES:}

[1] Fleischmann R, Jairath V, Mysler E et al. Nonmedical Switching From Originators to Biosimilars: Does the Nocebo Effect Explain Treatment Failures and Adverse Events in Rheumatology and Gastroenterology? Rheumatol Ther 7: 35-64 (2020).

Disclosure of Interests: None declared

DOI: 10.1136/annrheumdis-2021-eular.1563

\section{AB0219 EXPLORING LONG-TERM DRUG EFFECTIVENESS AND DRUG SURVIVAL FOR RITUXIMAB REFERENCE DRUG IN TREATMENT OF RHEUMATOID ARTHRITIS PATIENTS IN ORDINARY OUTPATIENT CLINIC}

K. Łosińska ${ }^{1}$, M. Wilk ${ }^{1}$, A. H. Pripp ${ }^{2}$, M. Korkosz ${ }^{1,3}$, G. Haugeberg ${ }^{4,5}$.

${ }^{1}$ University Hospital, Division of Rheumatology and Immunology, Cracow, Poland; ${ }^{2}$ Oslo University Hospital, Oslo Centre of Biostatistics and

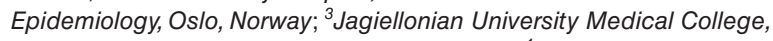
Department of Rheumatology, Cracow, Poland; ${ }^{4}$ Sorlandet Hospital, Division of Rheumatology, Department of Medicine, Kristiansand, Norway; ${ }^{5}$ NTNU, Norwegian University of Science and Technology, Department of Neuromedicine and Movement Science, Faculty of Medicine and Health Sciences, Trondheim, Norway

Background: In randomized controlled trials (RCTs) rituximab (RTX) has been shown to effectively suppress inflammation and reduce structural joint damage in rheumatoid arthritis (RA) patients [1]. There is a lack of RA data on RTX effectiveness and drug survival when used in real life practice.

Objectives: To explore long-term drug effectiveness and drug survival for RTX used to treat RA patients in ordinary clinical practice.

Methods: The study population included RA patients treated between 2006 and 2020 with RTX at an outpatient clinic in Norway. Patients were monitored using recommended measures for disease activity and patient reported out comes (PROs). Drug effectiveness was assessed with random intercept linear mixed models. Drug survival was described using Kaplan-Meier survival analysis. Baseline predictors of drug survival were assessed with multivariable Cox proportional hazard models.

Results: In database a total of 246 RA patients (females $74.8 \%$ ) were identified to have been treated with RTX. At baseline mean (SD) age was 59.1 (13.5) years, disease duration 13.0 (10.2) years, RF positive $88.8 \%$, ACPA positive $92.1 \%$. Majority of patients had first cycle RTX dosage of $2000 \mathrm{mg}$ $(82.9 \%)$. At baseline patients currently using conventional synthetic disease modifying anti-rheumatic drugs (csDMARDs) were $51.5 \%$ (methotrexate $39.4 \%$ ), prednisolone $73.6 \%$ and a total of $17.1 \%$ were biologic DMARDs (bDMARDs) naïve. In table 1 mean (SE) values for disease activity and PROs are shown for baseline and subsequent years after baseline for 5 years follow up.

Table 1. Changes in disease activity and PROs at baseline and subsequent 1 -year periods

\begin{tabular}{lcccccc}
\hline & $\begin{array}{c}\text { Baseline } \\
\mathrm{N}=246\end{array}$ & $\begin{array}{c}\text { 1 year } \\
\mathrm{N}=246\end{array}$ & $\begin{array}{c}2 \text { year } \\
\mathrm{N}=204\end{array}$ & $\begin{array}{c}3 \text { year } \\
\mathrm{N}=163\end{array}$ & $\begin{array}{c}4 \text { year } \\
\mathrm{N}=130\end{array}$ & $\begin{array}{c}5 \text { year } \\
\mathrm{N}=111\end{array}$ \\
\hline CRP (mg/L) & $23.1(2.1)$ & $21.3(1.6)$ & $11.7(0.9)$ & $8.6(1.4)$ & $6.2(0.7)$ & $6.7(0.9)$ \\
ESR (mm/hr) & $32.1(1.4)$ & $31.1(1.3)$ & $22.3(1.1)$ & $17.6(1.2)$ & $14.3(1.0)$ & $13.6(1.2)$ \\
SJC28 (0-28) & $6.3(0.4)$ & $5.4(0.3)$ & $3.2(0.3)$ & $2.2(0.2)$ & $1.6(0.2)$ & $1.5(0.2)$ \\
TJC28 (0-28) & $7.1(0.4)$ & $6.6(0.3)$ & $3.6(0.3)$ & $2.6(0.3)$ & $2.2(0.3)$ & $1.8(0.3)$ \\
DAS28 & $4.9(0.1)$ & $4.7(0.1)$ & $3.6(0.1)$ & $3.1(0.1)$ & $2.8(0.1)$ & $2.7(0.1)$ \\
CDAI & $22.9(0.9)$ & $20.7(0.7)$ & $12.3(0.7)$ & $9.4(0.7)$ & $8.5(0.6)$ & $7.7(0.7)$ \\
PGA (0-100mm) & $57.2(1.7)$ & $53.7(1.4)$ & $38.1(1.6)$ & $33.7(1.9)$ & $35.0(2.1)$ & $32.8(2.2)$ \\
MHAQ (0-3) & $1.0(0.0)$ & $0.9(0.0)$ & $0.7(0.0)$ & $0.6(0.0)$ & $0.5(0.0)$ & $0.5(0.0)$ \\
\hline
\end{tabular}

During follow up all disease activity and PRO measures improved significantly $(p<0.001)$. Least improvement was seen in first year and a more substantial improvement progressed since second year. Percentage of patients with no, moderate and good treatment response defined according to EULAR response criteria [2] is shown in figure 1. 


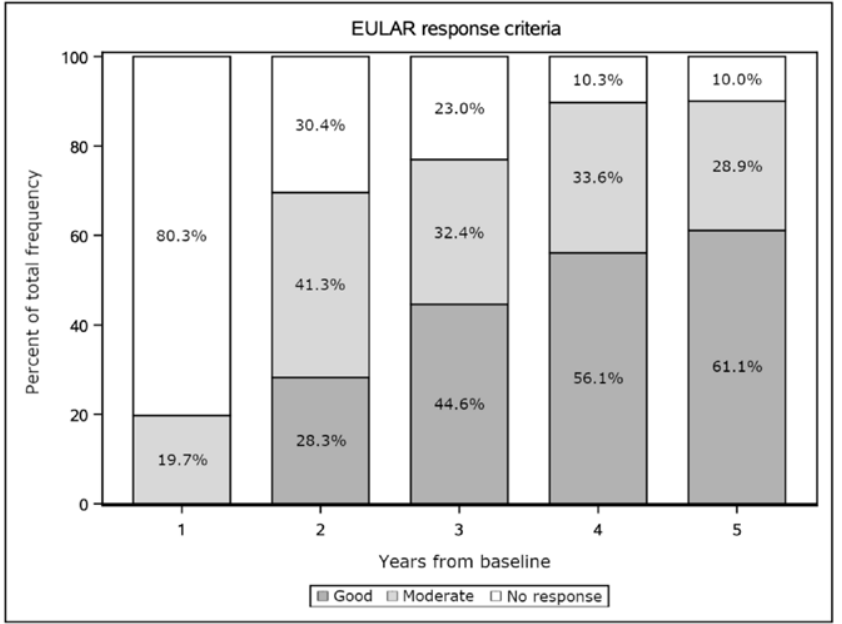

Figure 1. No, moderate and good response to RTX treatment

No significant difference during the 5-year follow-up was found regarding previous use of bDMARD or not and for concomitant use of csDMARDs or not for variables listed in the table 1.

Drug survival for the RTX was $83 \%(95 \mathrm{Cl} 77-87 \%)$ after 1 year, $66 \%(95 \mathrm{Cl}$ $60-72 \%)$ after 2 years, $53 \%(95 \mathrm{Cl} 46-59 \%)$ after 3 years, $46 \%(95 \mathrm{Cl} 39-52 \%)$ after 4 years and $34 \%(95 \mathrm{Cl} 28-40 \%)$ after 5 years of follow up.

No significant difference in drug survival was found between bDMARD naïve and previous users of bDMARDs or between concomitant and non-concomitant users of csDMARDs. RF positive patients had a better drug survival.

In prediction analysis RF positive status, high baseline DAS28, low baseline CRP, previous bDMARDs use, short disease duration and low MHAQ were found to be independently associated with better drug survival.

Conclusion: Our real life data shows that RTX treated RA patients had a satisfactory treatment response and drug survival declines rather linearly over time. However, a significant treatment response was achieved primary in the second year indicating that at least 2 twin infusions should be given before identifying treatment failure.

REFERENCES:

[1] Mok CC. Rituximab for the treatment of rheumatoid arthritis: An update. Drug Des Devel Ther 2013;8:87-100.

[2] Fransen J, van Riel PL. The Disease Activity Score and the EULAR response criteria. Rheum Dis Clin North Am. 2009;35:745-viii.

Acknowledgements: Nurses and doctors from Division of Rheumatology at Sørlandet Hospital in Kristiansand, Norway contributed to data collection. Disclosure of Interests: None declared DOI: 10.1136/annrheumdis-2021-eular.1661

\section{AB0220 ANTI-CITRULLINATED PROTEIN ANTIBODY (ACPA) POSITIVITY IS ASSOCIATED WITH REDUCED WITHDRAWAL RATES OF ABATACEPT IN RHEUMATOID ARTHRITIS BUT ONLY IN PATIENTS WHO ARE ANTI-NUCLEAR ANTIBODY (ANA) NEGATIVE}

J. Kimpton ${ }^{1}$, M. Shipa ${ }^{2}$, E. Hawkins ${ }^{2}$, S. A. Yeoh ${ }^{2}$, M. Greenwood ${ }^{3}$, M. Ehrenstein ${ }^{2} .{ }^{1}$ University College London, Centre for Clinical Pharmacology and Therapeutics, London, United Kingdom; ${ }^{2}$ University College London, Centre for Rheumatology, London, United Kingdom; ${ }^{3}$ University College London Hospital, Pharmacy Department, London, United Kingdom

Background: Abatacept, a selective inhibitor of T cell co-stimulation, is often used as a second-line biologic disease-modifying anti-rheumatic drug (bDMARD) after the failure of tumour necrosis factor inhibitor (TNFi) in Rheumatoid Arthritis (RA). However, in comparison to TNFi very few survival analyses of abatacept have been reported. ${ }^{1,2}$

Objectives: To investigate predictors of abatacept discontinuation due to either inefficacy or adverse events in RA patients over 5-years.

Methods: A retrospective observational analysis was conducted on a tertiary hospital dataset of RA (according to 2010 ACR/EULAR criteria) patients who started abatacept (either intravenous or subcutaneous). Time to abatacept discontinuation over 5-years was estimated using Kaplan-Meier survival analyses. A multivariate cox-regression model to predict abatacept discontinuation was chosen by elastic net regularisation.
Results: A total of 112 patients with RA [81\% female, mean age 58.1 (SD 13.5) years] commenced abatacept therapy during the study period. 88 (78.6\%) patients received intravenous abatacept, $14(15.9 \%)$ of whom switched to subcutaneous injection, and $24(21.4 \%)$ were initially treated with subcutaneous abatacept, $2(8.3 \%)$ of whom switched to intravenous. More than half of the patients $(65 / 112)$ were treated with at least one concomitant conventional synthetic DMARD (csDMARD). Methotrexate was the most commonly used $(n=37)$ csDMARD, followed by hydroxychloroquine $(n=23)$, sulfasalazine $(n=15)$, and leflunomide $(n=7) .42(37.5 \%)$ patients were treated with glucocorticoids (either oral, intra-articular, or intramuscular injection) during the time they were treated with abatacept. Abatacept was most commonly used as 4th $(n=29)$ and 3rd line $(n=24)$ bDMARD but 19 patients received abatacept as their first line bDMARD. $75(67 \%)$ patients were rheumatoid factor (RF) positive and $73(65.2 \%)$ were anti-citrullinated protein antibody (ACPA) positive. Anti-nuclear antibody (ANA) was positive in 32 patients. Abatacept was discontinued in 54 patients (48.2\%); $19(35.2 \%)$ due to an adverse event and 35 (64.8\%) due to loss of efficacy. Overall, the median time to discontinuation of abatacept was 3.8 years.

Multivariate cox regression (variables chosen by the elastic net and adjusted by whether or not abatacept was used as first-line therapy) showed that ACPA positivity was associated with a reduced risk of abatacept discontinuation with a hazard ratio (HR) of $0.40(95 \% \mathrm{Cl} 0.18$ to $0.85, \mathrm{p}=0.02, \mathrm{~N}$. of events $16 / 47)$ compared to ACPA negative patients (N. of events 15/23), but only if ANA was negative. In contrast, ACPA positivity did not reveal any retention benefit over ACPA negative patients, if they were ANA positive.

ACPA positive patients without positive ANA increased the time-to-discontinuation of abatacept predominantly after 3 -months (unadjusted log-rank $p=0.02$ ), compared to ACPA, and ANA negative patients (Figure 1). Adding csDMARDs with abatacept, reduced the risk of discontinuation of abatacept by $59 \%(95 \%$ $\mathrm{Cl} 24 \%$ to $77 \%, \mathrm{p}=0.004, \mathrm{~N}$. of events $26 / 65$ ) compared to monotherapy (N. of events 28/47).

Conclusion: Our data suggests patients who are ACPA positive and ANA negative are more likely to remain on abatacept therapy. Concomitant csDMARD use also acts as a positive predictor of abatacept treatment retention.

\section{REFERENCES:}

[1] Cagnotto G, et al. Arthritis Res Ther. 2020;22(1):15

[2] Alten R, et al. Clin Rheumatol. 2019;38(5):1413-1424.

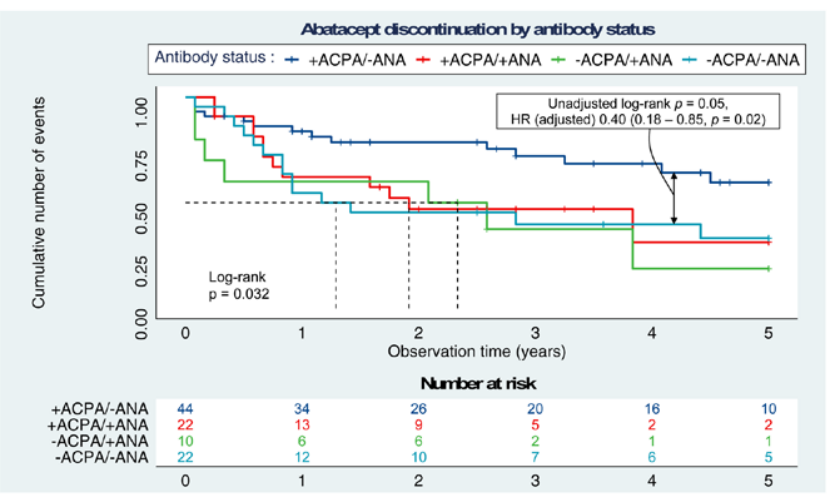

Figure 1. Kaplan-Meier survival curve of retention of abatacept, stratified by Anti-citrullinated protein antibody (ACPA) and antinuclear antibody (ANA).

$+A C P A /-A N A=A C P A$ positive and ANA negative, $+A C P A /+A N A=A C P A$ positive and $A N A$ positive, $-A C P A /-A N A=A C P A$ negative and $A N A$ negative, $-A C P A /+A N A=A C P A$ negative and $A N A$ positive, $H R=$ Hazard ratio.

Acknowledgements: I have no acknowledgements to declare.

Disclosure of Interests: None declared

DOI: 10.1136/annrheumdis-2021-eular.1848

\section{\begin{tabular}{|l|l}
\hline AB0221 CURRENT IMPLEMENTATION OF TREAT-TO-TARGET \\
\hline
\end{tabular} APPROACH IN RHEUMATOID ARTHRITIS TREATMENT: THE PERSPECTIVE OF CHINESE RHEUMATOLOGISTS}

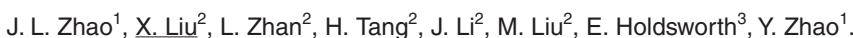
${ }^{1}$ Peking Union Medical College Hospital, Department of Rheumatology, Beijing, China; ${ }^{2}$ Eli Lilly and Company, Medical, Shanghai, China; ${ }^{3}$ Adelphi Real World, Rheumatology, Manchester, United Kingdom

Background: Treat-to-target (T2T) approach is recommended as a standard management strategy in rheumatoid arthritis (RA) treatment by Chinese guideline for diagnosis and treatment of $\mathrm{RA}^{[1]}$. However, there is little known about its current implementation in China. 